\title{
Imaging of Biomedical Data Using a Multiplicative Regularized Contrast Source Inversion Method
}

\author{
Aria Abubakar, Peter M. van den Berg, and Jordi J. Mallorqui, Member, IEEE
}

\begin{abstract}
In this paper, the recently developed multiplicative regularized contrast source inversion method is applied to microwave biomedical applications. The inversion method is fully iterative and avoids solving any forward problem in each iterative step. In this way, the inverse scattering problem can efficiently be solved. Moreover, the recently developed multiplicative regularizer allows us to apply the method blindly to experimental data. We demonstrate inversion from experimental data collected by a 2.33-GHz circular microwave scanner using a two-dimensional (2-D) TM polarization measurement setup. Further some results of a feasibility study of the present inversion method to the 2-D TE polarization and the full-vectorial three-dimensional measurement will be presented as well.
\end{abstract}

Index Terms-Experimental data, medical applications, microwave tomography, nonlinear inversion, three-dimensional.

\section{INTRODUCTION}

$\mathbf{M}$ ICROWAVE imaging techniques for biomedical applications are much less developed than those based on ultrasound, X-rays, nuclear magnetic imaging, or even electrical impedance tomography. Standard diffraction tomography methods have been shown to offer limited capabilities in terms of quantitative reconstructions of the complex permittivity [1]. Fortunately, in the last decade, microwave tomography has made a significant step with the development of various iterative reconstruction algorithms providing serious expectations in obtaining quantitative images (see [2]-[5]). Particularly, the ability to handle the high complex permittivity targets marked a sharp difference with previous standard diffraction tomography algorithms. The latter algorithms provided only qualitative results, namely, the equivalent current distribution in the target. On the other hand, the quantitative algorithms aim to a reconstruction of the complex permittivity of the target delivered from the local field dependence involved in the equivalent current distribution. As is well known, the price to pay is to solve a nonlinear inverse problem, instead of a linear one which can computationally be (very) expensive. Due to this expensive computation requirement, the use of a noniterative algorithm will not be feasible.

The most popular quantitative reconstruction algorithm used for biomedical applications is the Newton-Kantorovich technique which aims to minimize iteratively the error between the

Manuscript received May 8, 2001.

A. Abubakar and P. M. van den Berg are with the Centre for Technical Geoscience, Delft University of Technology, 2628 CD Delft, The Netherlands (e-mail: abubakar@its.tudelft.nl).

J. J. Mallorqui is with the Department of Signal Theory and Communications, Universitat Politecnica de Catalunya, 08034 Barcelona, Spain.

Publisher Item Identifier 10.1109/TMTT.2002.800427. measured scattered field by the targets and the scattered field calculated from a numerical model (see [6] and [7]). In this method, one has to solve the multiview forward problem in each iterative step. This aspect will limit the applicability of the method for the full-vectorial three-dimensional (3-D) case. Another type of algorithm which avoids solving any forward problem in each iterative step is introduced by van den Berg and Kleinman [2] for the two-dimensional (2-D) case and extended by Abubakar and van den Berg [8] to the full-vectorial 3-D static case. In this method, the complex permittivity contrast and the contrast sources (the product of the complex permittivity contrast with the total field) are iteratively reconstructed by minimizing a cost functional using conjugate gradient directions. Recently, the method has been modified to include a multiplicative regularization factor by van den Berg et al. [9] and extended by Abubakar et al. [10]. The multiplicative technique allows the method to use a regularization factor without the necessity of determining an artificial weighting parameter. This regularization parameter is determined by the iterative process itself, which makes the method suitable to invert experimental data as shown by Bloemenkamp et al. [11].

In this paper, the improved version of the method, the so-called multiplicative regularized contrast source inversion (MR-CSI) method, described in [12], has been modified to carry out inversion of microwave biomedical data. Further feasibility study results of the applications of the MR-CSI method in 2-D TE-polarization and full-vectorial 3-D cases (which up to now is known as a difficult case to handle [13]) are presented. This paper is organized as follows. First, the integral representations and the inversion algorithm will be described. Then, for the 2-D TM-polarization case, inversion results of some experimental data collected at UPC Barcelona using a circular microwave scanner at $2.33 \mathrm{GHz}$ developed by Broquetas et al. [14] will be presented. After that, some comparisons of inversion of synthetic data for 2-D TM- and 2-D TE-polarization measurement will be discussed. Then, as a final example, we present the inversion results of a simple 3-D model using full-vectorial measurements.

\section{INTEGRAL REPRESENTATIONS}

Let an incident electric field $\boldsymbol{E}^{\text {inc }}$ illuminate an inhomogeneous dielectric object $B$ of complex permittivity $\epsilon(\boldsymbol{x})$ and arbitrary shape. The complex permittivity and the shape of this object is unknown, but they are known to lie within a bounded simply connected object domain $D$ (see Fig. 1). This object domain $D$ is assumed to be embedded in a known background medium of complex permittivity $\epsilon_{b}$. For this biomedical application, the background medium is known and is homogeneous. 


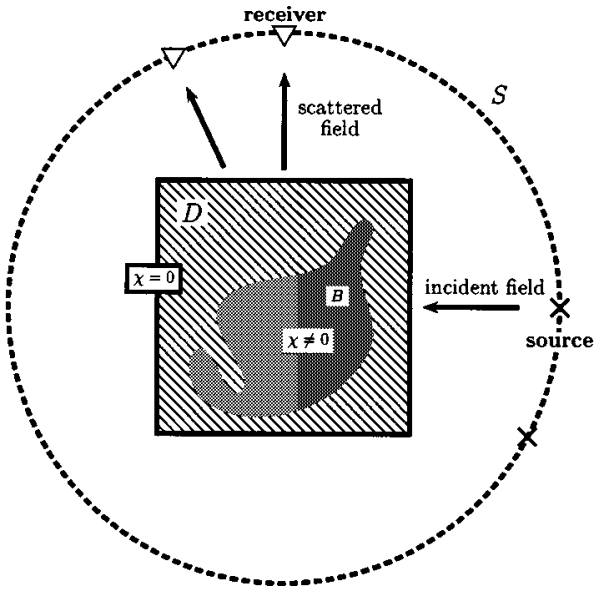

Fig. 1. Geometrical model for the scattering experiment.

The position vector is denoted by $\boldsymbol{x}$. We assume a time-harmonic dependence $\exp (j \omega t)$, where $j^{2}=-1, \omega$ is angular frequency, and $t$ is time.

\section{A. 2-D TM-Polarization Case}

For 2-D TM polarization, the electric field is parallel to objects. In our case, $\boldsymbol{E}=E_{3}(\boldsymbol{x}) \boldsymbol{i}_{3}$. We then deal with a scalar problem. The quantity $E_{3}^{\text {inc }}(x)$ denotes an incident electric field due to a line source located at $\boldsymbol{x}^{S}$ with polarization in the $\boldsymbol{i}_{3}$ direction parallel to the cylindrical objects. The line sources are located in a data domain $S$ surrounding the object domain $D$, where the measurements are made as well, $x^{R} \in S$. Then, for this 2-D TM polarization, the scattered field can be represented in an integral form

$$
E_{3}^{\mathrm{sct}}\left(\boldsymbol{x}^{R}\right)=k_{b}^{2} \int_{D} g\left(\boldsymbol{x}^{R}-\boldsymbol{x}^{\prime}\right) \chi(\boldsymbol{x}) E_{3}(\boldsymbol{x}) d v\left(\boldsymbol{x}^{\prime}\right)
$$

where the 2-D Green's function $g$ is given by

$$
g(\boldsymbol{x})=\frac{-j}{4} H_{0}^{(2)}\left(k_{b}|\boldsymbol{x}|\right), \quad k_{b}=\omega \sqrt{\mu_{0} \epsilon_{b}}
$$

in which $H_{0}^{(2)}$ is the zeroth-order Hankel function of the second kind and $\mu_{0}$ is the permeability in vacuum. In (1), the contrast $\chi$ is given by

$$
\chi(\boldsymbol{x})=\frac{\epsilon(\boldsymbol{x})-\epsilon_{b}}{\epsilon_{b}}
$$

where

$$
\epsilon(\boldsymbol{x})=\epsilon_{0}\left[\epsilon_{r}^{\prime}(\boldsymbol{x})+j \epsilon_{r}^{\prime \prime}(\boldsymbol{x})\right]=\epsilon_{0}\left[\epsilon_{r}(\boldsymbol{x})-j \frac{\sigma(\boldsymbol{x})}{\omega \epsilon_{0}}\right]
$$

in which $\epsilon_{r}(\boldsymbol{x})$ and $\sigma(\boldsymbol{x})$ are the relative permittivity and the conductivity, and $\epsilon_{0}$ is the permittivity of vacuum. The total field $E_{3}$ inside the object domain $D$ is known to satisfy the domain integral equation

$$
E_{3}^{\mathrm{inc}}(\boldsymbol{x})=E_{3}(\boldsymbol{x})-k_{b}^{2} \int_{D} g\left(\boldsymbol{x}-x^{\prime}\right) \chi\left(\boldsymbol{x}^{\prime}\right) E_{3}\left(\boldsymbol{x}^{\prime}\right) d v\left(\boldsymbol{x}^{\prime}\right) .
$$

Equations (1) and (5) are the two basic equations to develop the inversion procedure for the 2-D TM-polarization case.

\section{B. 2-D TE-Polarization Case}

For 2-D TE polarization, the electric field has two components in the transversal plane of the objects. In our case, $\boldsymbol{E}=$ $E_{1} \boldsymbol{i}_{1}+E_{2} \boldsymbol{i}_{2}$, while $\boldsymbol{E}^{\text {inc }}$ denotes the incident electric field due to a line source with polarization in the transversal plane located at $\boldsymbol{x}^{S}$. The line sources are located in a data domain $S$ surrounding the object domain $D$, where the measurements are made as well, $\boldsymbol{x}^{R} \in S$. Then, for this 2-D TE polarization, the scattered field can be represented in an integral form

$$
\boldsymbol{E}^{\mathrm{sct}}\left(\boldsymbol{x}^{R}\right)=\left(k_{b}^{2}+\nabla^{R} \nabla^{R} \cdot\right) \int_{D} g\left(\boldsymbol{x}^{R}-\boldsymbol{x}^{\prime}\right) \chi(\boldsymbol{x}) \boldsymbol{E}(\boldsymbol{x}), d v\left(\boldsymbol{x}^{\prime}\right)
$$

where $\nabla^{R}$ is the spatial differentiation operator with respect to $\boldsymbol{x}^{R}$. In this 2-D TE polarization, only the scattered electric field tangential to the receiver aperture is measured. We then require the integral representation in (6) to be satisfied for this tangential component. This measurement setup is similar to the one used by Bloemenkamp et al. [11] for the 2-D TE-polarization case. The total field inside the object domain $D$ is known to satisfy the domain integral equation

$$
\boldsymbol{E}^{\mathrm{inc}}(\boldsymbol{x})=\boldsymbol{E}(\boldsymbol{x})-\left(k_{b}^{2}+\nabla \nabla \cdot\right) \int_{D} g\left(\boldsymbol{x}-\boldsymbol{x}^{\prime}\right) \chi\left(\boldsymbol{x}^{\prime}\right) \boldsymbol{E}\left(\boldsymbol{x}^{\prime}\right) d v\left(\boldsymbol{x}^{\prime}\right) .
$$

Equations (6) and (7) are the two basic equations to develop the inversion procedure for the 2-D TE-polarization case.

\section{3-D Full-Vectorial Case}

In a 3-D full-vectorial problem, the electric incident $\boldsymbol{E}^{\text {inc }}$ field is a three-components vector. For simplicity, we consider a point magnetic dipole source directed in the vertical direction, the $\boldsymbol{i}_{3}$ direction, located at $\boldsymbol{x}^{S}$. This magnetic dipole source is located in a data domain $S$ surrounding the object domain $D$, where the measurement $x^{R} \in S$ is made as well. Then, for this 3-D full-vectorial case, the scattered field can be represented in an integral form

$$
\boldsymbol{E}^{\mathrm{sct}}\left(\boldsymbol{x}^{R}\right)=\left(k_{b}^{2}+\nabla^{R} \nabla^{R} \cdot\right) \int_{D} G\left(\boldsymbol{x}^{R}-\boldsymbol{x}^{\prime}\right) \chi(\boldsymbol{x}) \boldsymbol{E}(\boldsymbol{x}) d v\left(\boldsymbol{x}^{\prime}\right)
$$

where the 3-D Green's function $G$ is given by

$$
G(\boldsymbol{x})=\frac{\exp \left[-j k_{b}|\boldsymbol{x}|\right]}{4 \pi|\boldsymbol{x}|} .
$$

In this full-vectorial 3-D case, we measure all the components of the scattered electric field. The total field inside the object domain $D$ is known to satisfy the domain integral equation

$$
\boldsymbol{E}^{\mathrm{inc}}(\boldsymbol{x})=\boldsymbol{E}(\boldsymbol{x})-\left(k_{b}^{2}+\nabla \nabla \cdot\right) \int_{D} G\left(\boldsymbol{x}-\boldsymbol{x}^{\prime}\right) \chi\left(\boldsymbol{x}^{\prime}\right) \boldsymbol{E}\left(\boldsymbol{x}^{\prime}\right) d v\left(\boldsymbol{x}^{\prime}\right) .
$$

Equations (8) and (10) are the two basic equations to develop the inversion procedure for the full-vectorial 3-D case. 


\section{INVERSE ALGORITHM}

In order to discuss our solution of the inverse scattering problem, we first write our basic equations in an operator notation. We denote either the scalar electric field for the 2-D TM-polarization case, the two-components electric field vector for the 2-D TE-polarization case, or the three-components electric field vector for the full-vectorial 3-D case by the symbol $u$. The data quantities either the scalar scattered electric field, the two-component scattered electric field vector, or the three-component vector scattered electric field vector are denoted by $f$.

We assume that the unknown object is illuminated successively by a number of known incident fields $u_{i}^{\text {inc }}, i=1, \ldots, I$. For each incident field $u_{i}^{\text {inc }}$, the total field will be denoted by $u_{i}$ and the measured scattered field data are denoted by $f_{i}$. Then, the integral representations of the measurement data in (1), (6), and (8) are written as

$$
f_{i}=G_{S} \chi u_{i}, \quad x \in S
$$

while the domain integral equations in (5), (7), and (10) are written as

$$
u_{i}=u_{i}^{\mathrm{inc}}+G_{D} \chi u_{i}, \quad \boldsymbol{x} \in D .
$$

The profile reconstruction problem is that of finding $\chi$ of the object domain $D$ for given $f_{i}$ at the data domain $S$, or solving the data equation in (11) for $\chi$, subject to the additional condition that $\chi$ and $u_{i}$ on $D$ satisfy (12). This problem is nonlinear and has to be solved iteratively.

We observed that the data equations contain the unknown fields inside the scattering object and the contrast in the form of a product; it can be written as a single quantity, viz. the contrast sources

$$
w_{i}(\boldsymbol{x})=\chi(\boldsymbol{x}) u_{i}(\boldsymbol{x})
$$

which can be considered as equivalent sources that produce the measured scattered fields. Using the definition of the contrast sources in (13), we obtained the data equations in terms of the contrast sources as follows:

$$
f_{i}=G_{S} w_{i}, \quad x \in S .
$$

Multiplying both sides of (12) with $\chi$, and using (13), we define in symbolic form the object equations as

$$
w_{i}=\chi u_{i}^{\text {inc }}+\chi G_{D} w_{i}, \quad \boldsymbol{x} \in D .
$$

We consider (14) and (15) as two equations from which we want to determine the unknown contrast $\chi$ and the unknown contrast sources $w_{i}$ in $D$.

The MR-CSI method constructs alternatively sequences of contrast sources $w_{i, n}$ for $(n=1,2, \ldots)$ and the contrast $\chi_{n}$ for $(n=1,2, \ldots)$ by minimizing a cost functional

$$
C_{n}\left(w_{i}, \chi\right)=F_{n}\left(w_{i}, \chi\right) F_{n}^{R}(\chi)
$$

where

$$
F_{n}\left(w_{i}, \chi\right)=F^{S}\left(w_{i}\right)+F_{n}^{D}\left(w_{i}, \chi\right)
$$

in which

$$
\begin{aligned}
F^{S}\left(w_{i}\right) & =\eta^{S} \sum_{i}\left\|f_{i}-G_{S} w_{i}\right\|_{S}^{2} \\
F_{n}^{D}\left(w_{i}, \chi\right) & =\eta_{n}^{D} \sum_{i}\left\|\chi u_{i}^{\text {inc }}-w_{i}+\chi G_{D} w_{i}\right\|_{D}^{2} .
\end{aligned}
$$

The normalization factors are chosen as

$$
\begin{aligned}
\eta^{S} & =\left(\sum_{i}\left\|f_{i}\right\|_{S}^{2}\right)^{-1} \\
\eta_{n}^{D} & =\left(\sum_{i}\left\|\chi_{n-1} u_{i}^{\mathrm{inc}}\right\|_{D}^{2}\right)^{-1} .
\end{aligned}
$$

In (16) $F_{n}^{R}$ is the weighted $L^{2}(D)$-norm total variation regularization factor

$$
F_{n}^{R}(\chi)=\frac{1}{V} \int_{D} \frac{|\nabla \chi(\boldsymbol{x})|^{2}+\delta_{n-1}^{2}}{\left|\nabla \chi_{n-1}(\boldsymbol{x})\right|^{2}+\delta_{n-1}^{2}} d v(\boldsymbol{x})
$$

where $V=\int_{D} d v(\boldsymbol{x})$ denotes the volume of the object domain $D$. We have included the regularization factor $F_{n}^{R}$ as a multiplicative constraint, with the result that the cost functional $F_{n}$ is the weighting parameter, i.e., determined by the inversion problem itself. This eliminates the choice of the artificial regularization parameters completely. In (22), $\delta_{n-1}^{2}$ is chosen as

$$
\delta_{n-1}^{2}=F_{D, n-1} \tilde{\Delta}^{2}
$$

where $\tilde{\Delta}$ denotes the reciprocal mesh size of the discretized domain $D$ and $F_{n-1}^{D}$ is the normalized error in the object equation of the previous iteration, cf. (19).

The cost functional in (16) is based on two things: the objective of minimizing the errors in the data and object equations and the observation that the regularization factor, when minimized, converges to one. The structure of the cost functional is such that it will minimize the regularization factor $F_{n}^{R}$ with a large weighting parameter in the beginning of the optimization process, because the value of $F_{n}$ is still large, and that it will gradually minimize more and more the errors in the data and object equations when the regularization factor has reached a nearly constant value close to one. If noise is present in the data, the errors in the data equations will remain at a large value during the optimization and, therefore, the weight of the regularization factor will be more significant. Hence, the noise will, at all times, be suppressed in the reconstruction process and we automatically fulfill the need of a stronger regularization when the data contain noise.

The factor $\delta_{n-1}^{2}$ is introduced for restoring differentiability. Its choice is further inspired by the idea that, in the first few iterations, we do not need the minimization of the regularization factor and when the iterations proceed we want to increase the effect of the regularization factor.

This MR-CSI algorithm starts with some initial estimates obtained from the back propagation. Then, in each iteration, it reconstructs alternatingly the contrast sources and the contrast by using conjugate gradient steps (one for the contrast sources and one for the contrast). In this way, the computational complexity of the algorithm is approximately equal of solving two forward 


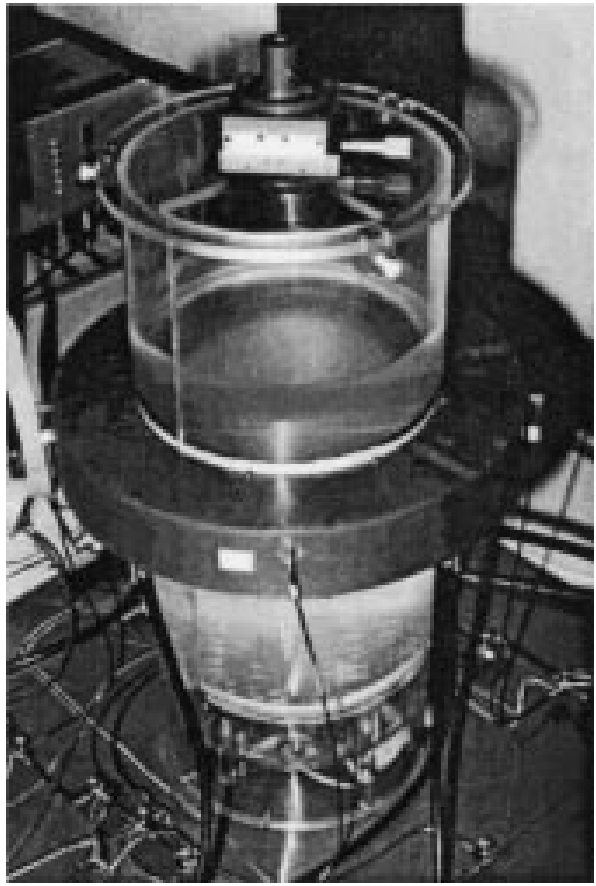

Fig. 2. Photograph of the $2.33-\mathrm{GHz}$ circular scanner.

problems using conjugate gradient method. The details of the algorithm can be found in the Appendix.

In biomedical applications, we have some a priori information about the range of the complex permittivity $\epsilon(\boldsymbol{x})$. We remark that this a priori information is easily implemented by enforcing, after each update of the contrast, the complex permittivity beyond its range to either the lower or the upper bound. In most numerical examples presented later in this paper, we have used this a priori information.

\section{EXPERIMENTAL DATA: 2-D TM CASE}

For the 2-D TM-polarization case, there are experimental data available. The circular microwave scanner with frequency of operation at $2.33 \mathrm{GHz}$ is used. It consists of a $12.5-\mathrm{cm}$-radius circular array of 64 water-immersed horn antennas, see [13]. The electric field is parallel to the array axis, the $i_{3}$ axis. The targets are introduced in a cylindrical water tank (see Fig. 2). Each of the array antennas can operate either in a transmitting or receiving mode. The measurement procedure records the total electric field values at the receiving antennas, when all the array antennas are successively used as a transmitter. Due to isolation limitations of the circuitry, if one antenna is transmitting, the scattered fields are measured only with the 33 antennas located in front of the active source. A schematic measurement setup of the antenna array is given in Fig. 3. The scattered fields are deduced from the total field by subtracting the incident field, measured in the absence of any targets. Furthermore, the measured scattered fields have been calibrated so that a unit line source directed in the $\boldsymbol{i}_{3}$ direction can be used as the model of the incident fields viz.

$$
E_{i}^{\mathrm{inc}}(\boldsymbol{x})=-\frac{\omega \mu_{0}}{4} H_{0}^{(2)}\left(k_{b}\left|\boldsymbol{x}-\boldsymbol{x}_{j}^{S}\right|\right)
$$

where $\mu_{0}=4 \pi \times 10^{-7}$ is the permeability in vacuum.

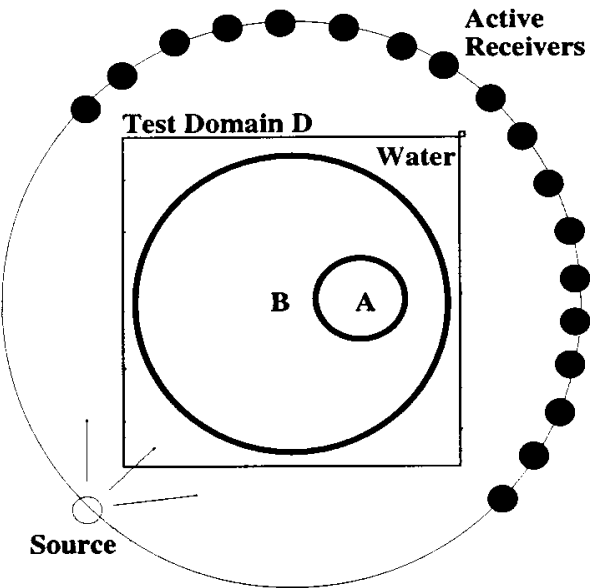

Fig. 3. Measurement setup of the experimental data and the cylindrical phantom (schematic).

In the inversion of experimental data, we assumed that the unknowns object entirely located within a test domain $D$ with dimension of $6.4 \lambda$ by $6.4 \lambda$. The quantity $\lambda$ is the wavelength in water with $\epsilon_{b}=77.3-j 8.66$ at frequency $f=2.33 \mathrm{GHz}$. Hence, the wavelength $\lambda=14.6 \mathrm{~mm}$. The discrete form of the algorithm is obtained by dividing the test domain into 64 by 64 subsquares, assuming the contrast, the contrast sources, and the fields to be piecewise constant. The integrals over subsquares are approximated by integrals over circles of equal area which are calculated analytically [15]. The discrete spatial convolutions are efficiently computed using fast Fourier transform (FFT) routines.

The lower and upper bounds of the complex permittivity used in the inversion algorithm are given by

$$
0 \leq \epsilon_{r}^{\prime}(\boldsymbol{x}) \leq 80 \text { and } 0 \leq-\epsilon_{r}^{\prime \prime}(\boldsymbol{x}) \leq 20 .
$$

\section{A. Cylindrical Phantom}

The first experimental data (data file: FANCENT.ASC) were obtained from a phantom consisting of two Plexiglas cylinders, filled with different concentrations of ethyl alcohol. Fig. 3 shows the phantom for which the measurements were taken. Cylinder A was filled with a $96 \%$ solution of ethyl alcohol, with $\epsilon=10-j 8.3$. Cylinder B was filled with a $4 \%$ ethyl alcohol solution, with $\epsilon=73-j 11$. The complex permittivity of the Plexiglas was $\epsilon=2.73-j 0.01$.

The results obtained from the initial estimates (back propagation) are given in the top plots in Fig. 4. This is approximately identical if we use the spectral diffraction tomography technique. From the back propagation results, we can "already" observe the presence of cylinder B, but the indication of the presence of cylinder A is very poor. Nevertheless, the reconstructed values are completely wrong.

The results of our nonlinear inversion method described in the present paper after 1024 iterations are given in the bottom plots of Fig. 4. Although the total number of iterations is large, the total computation time is limited. Note that we do not solve any forward problem in each iteration of the algorithm. One iteration of the method takes approximately $8 \mathrm{~s}$ on a personal computer with a $600-\mathrm{MHz}$ Pentium III processor. After 1024 iterations, 

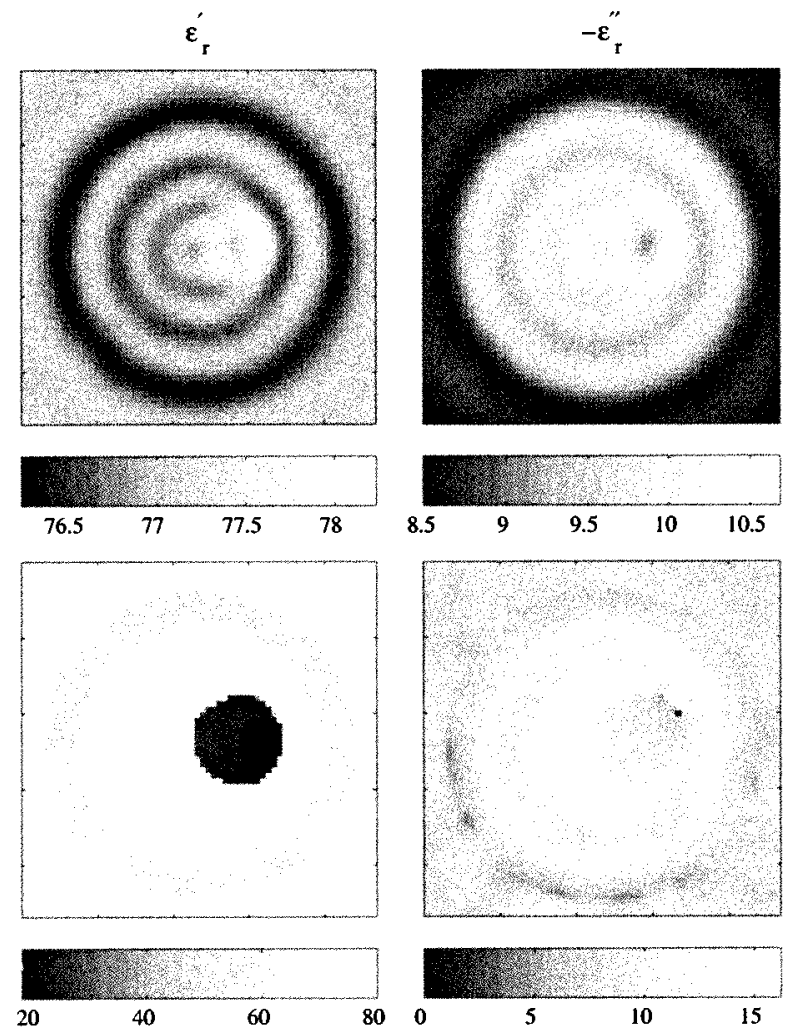

Fig. 4. Cylindrical phantom: images obtained from back propagation (top plots) and from nonlinear inversion (bottom plots).

the normalized error $F_{n}^{S}$ in the data equations is already reduced to $4.08 \%$, and adding more iterations does not change the result.

Note that this particular experimental data has also been used to test the Newton-Kantorovich method by Joachimiwicz et al. [7]. They have obtained a comparable numerical result, but in the Newton-Kantorovich method more a priori information, like the location of the boundary of cylinder B and its (approximate) permittivity value, has to be used. Without using this $a$ priori information, the Newton-Kantorovich method was not able to produce acceptable results [7].

\section{B. Human Arm Phantom}

The next experimental data (data file: PHANARM.CO) were obtained from a human arm phantom. The external layer (skin) and bones of the human arm phantom were made with PVC with complex permittivity $2.73-j 0.01$ and the muscle was $54.5-$ j17.2. Again we show first the results obtained from the initial estimates (back propagation). These results are given in the top plots of Fig. 5. The results of our nonlinear inversion method after 1024 iterations are given in the bottom plots of Fig. 5. After 1024 iterations, the normalized error $F_{n}^{S}$ in the data equations is already reduced to $6.40 \%$, and adding more iterations does not change the result. From the results, we observe that the bones are clear and sharp. The drawback is that for one of the bones the imaginary part of the reconstructed complex permittivity is completely wrong. This can be caused by the presence of the noise in the experimental data (in typical operational conditions the SNR is around $20 \mathrm{~dB}$ ).
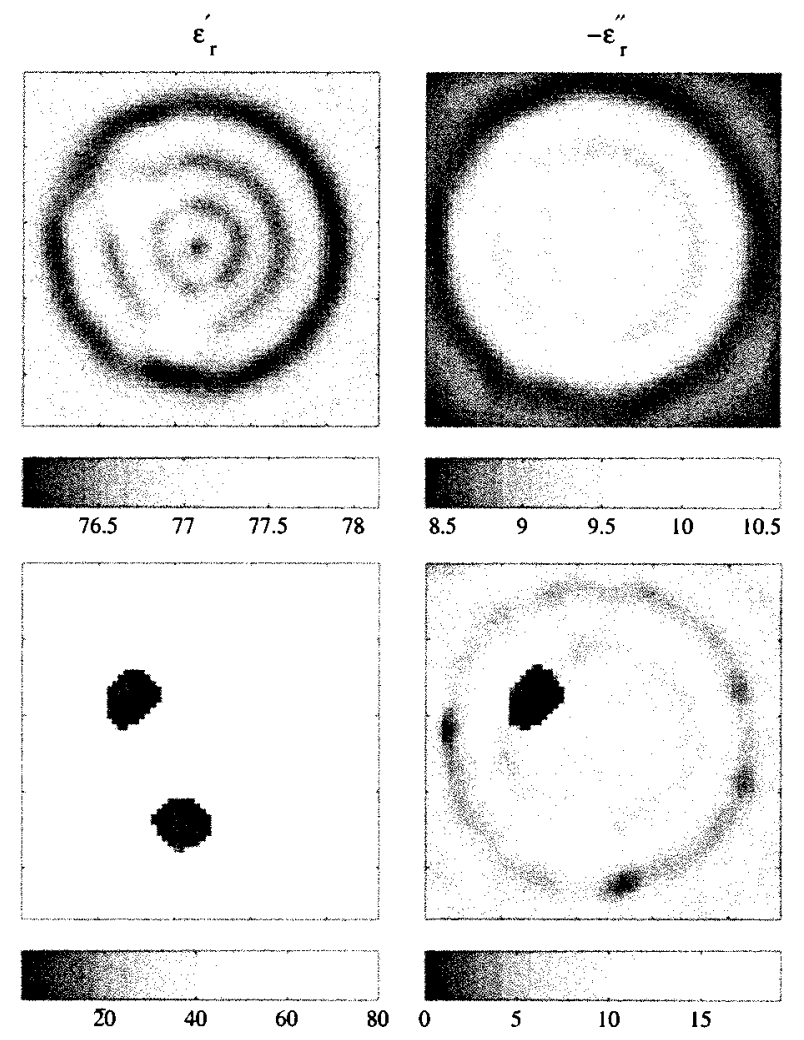

Fig. 5. Human arm phantom: images obtained from back propagation (top plots) and from nonlinear inversion (bottom plots).

\section{Human Forearm}

As the last experimental data, we consider data that were taken from a human forearm (data-file: BRAGREG.ASC). The back propagation results are given in the top plots of Fig. 6. The results of our nonlinear inversion method after 1024 iterations are given in the middle plots of Fig. 6. After 1024 iterations, the normalized error $F_{n}^{S}$ in the data equations is already reduced to $4.10 \%$, and adding more iterations does not change the result. The reconstructed images show the positions of the two bones and the correct value of the muscle (approximately 54.5 - j17.2). Conversely, due to the water and tissue attenuation and the reduced dynamic range of the available data, the complex permittivity values of the bones are higher than the real ones (the value should approximately be $5.5-j 0.59$ at $2.33 \mathrm{GHz}$ ).

In order to investigate whether we can improve the results by reducing the cell size, in the bottom plots of Fig. 6 we present also inversion results using a discretization mesh that is twice as fine. Thus, now the test domain $6.4 \lambda$ by $6.4 \lambda$ is discretized into 128 by 128 cells. The computation time is approximately increased by a factor of four. We observe that the complex permittivity values of the reconstructed bones are improved slightly. The normalized error $F_{n}^{S}$ in the data equations is now reduced to $4.06 \%$, which is lower than the previous value. Thus, for this particular data set, reducing the mesh size is not advantageous. 

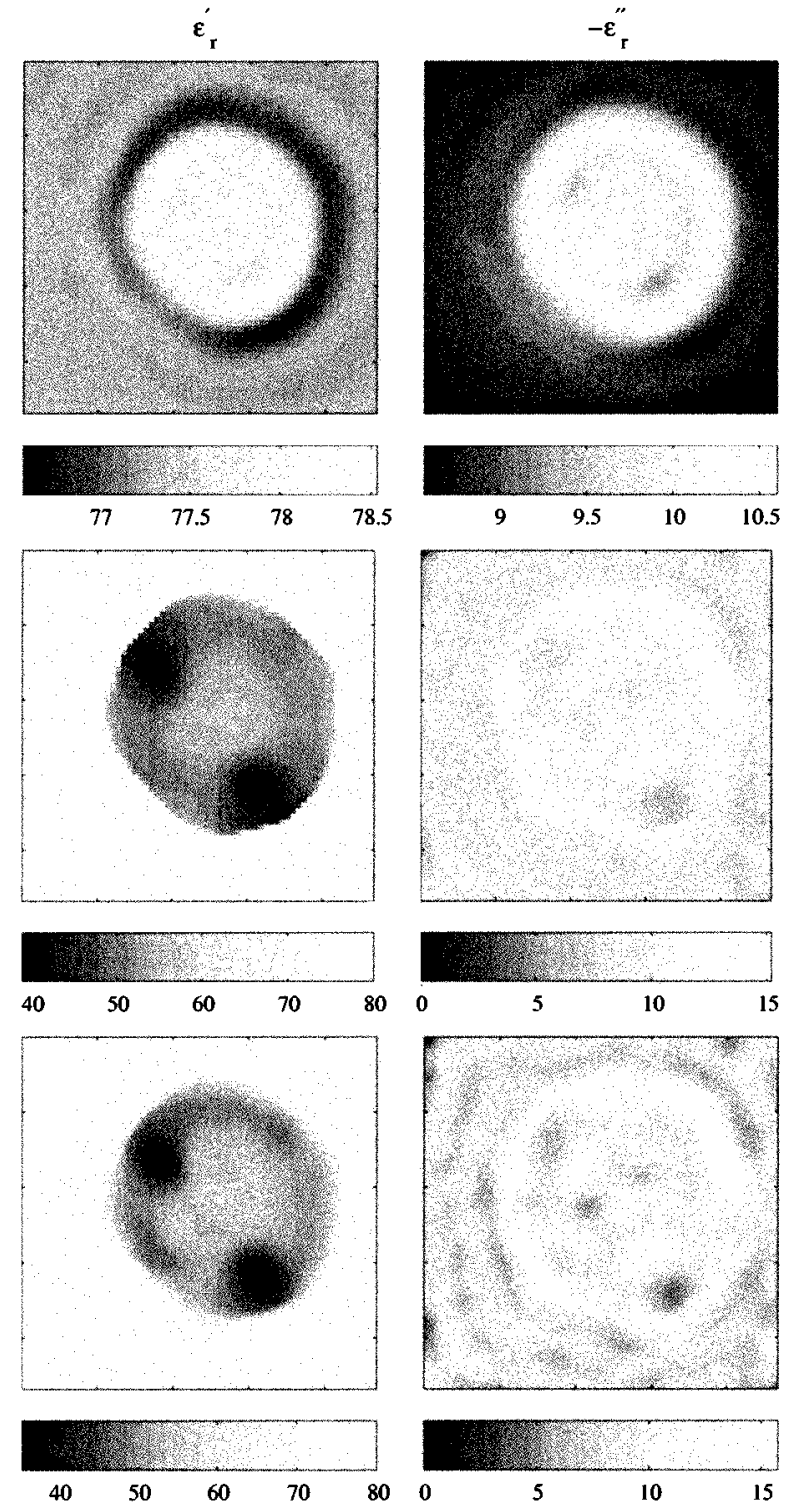

Fig. 6. Human forearm: images obtained from back propagation (top plots), from nonlinear inversion using 64 by 64 cells (middle plots) and using 128 by 128 cells (bottom plots).

\section{COMPARISON OF 2-D TM- AND 2-D TE-POLARIZATION MEASUREMENTS}

In this section, we present some comparisons of the inversion using 2-D TM- and 2-D TE-polarization measurements. Because there are no experimental data available for the 2-D TE-polarization case, we use synthetic data. The 2-D TE-polarization measurements can be obtained by rotating the horn antennas over 90 degrees in the circular measurement antennas. The synthetic data are generated using a Conjugate Gradient FFT method; see [15] for 2-D TM-polarization measurements and [16] for 2-D TE-polarization measurements. Note that, due to the gradient-divergence operator working on the integral over domain $D$, the discretization procedure of the TE-polarization measurements is more complicated than that for the TM-polarization measurements. After generation of synthetic data, 5\% random additive white noise of the maximum value of all the scattered field are added to the data. In the inversion algorithm
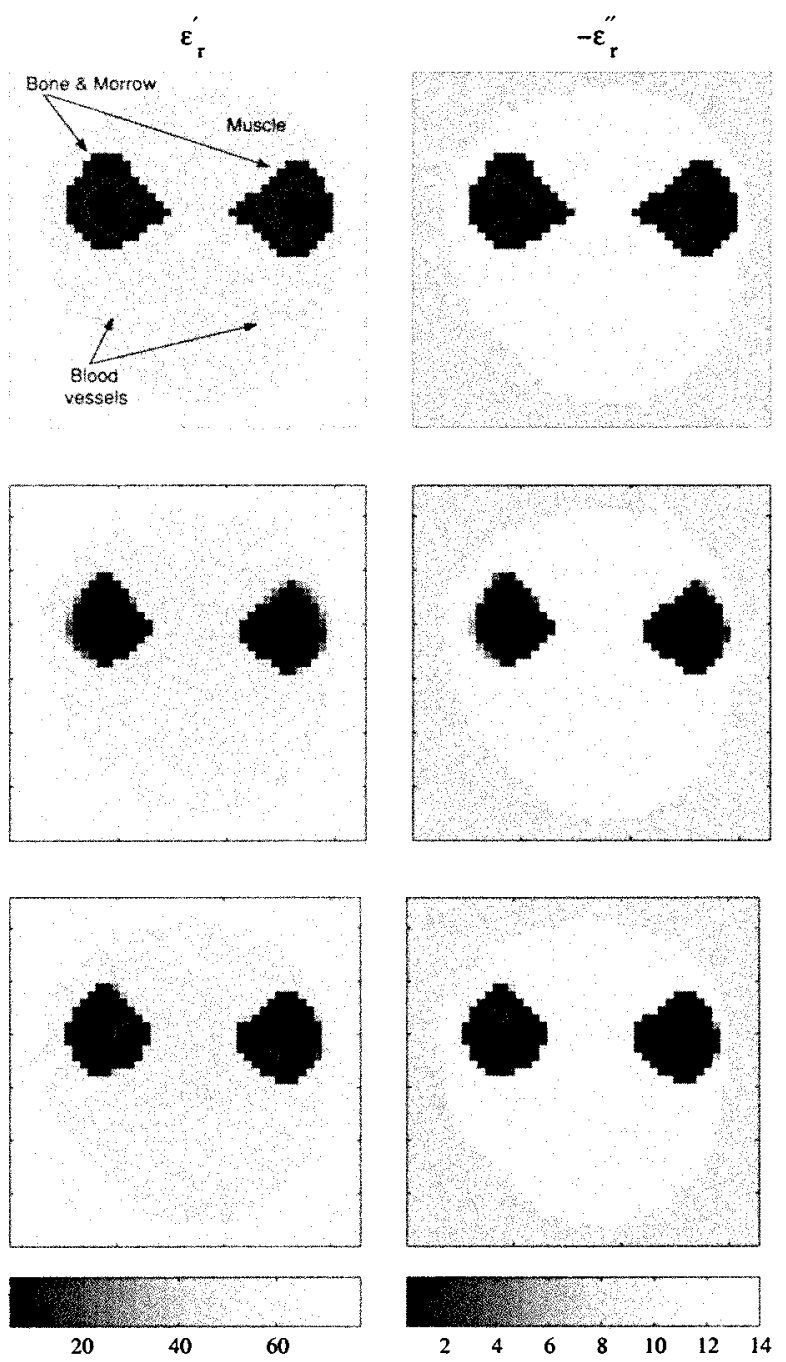

Fig. 7. Synthetic arm: images of the original profile (top plots) and the results using 2-D TM polarization (middle plots), and using 2-D TE polarization (bottom plots)

of the 2-D TE-polarization measurement, we use a slightly different technique (see [17]) to obtain the discrete form of the inversion algorithm than the one used to generate synthetic data.

In order to have a criterion of the quality of the reconstructed images, we define the contrast error as follows:

$$
\mathrm{ERR}_{n}=\sqrt{\frac{\left\|\epsilon(\boldsymbol{x})-\epsilon^{\text {orig }}(\boldsymbol{x})\right\|_{D}^{2}}{\left\|\epsilon^{\text {orig }}(\boldsymbol{x})\right\|_{D}^{2}}}
$$

where $\epsilon^{\text {orig }}(x)$ is the original profile which has been used to generate synthetic data. Note that for the study using the synthetic data the a priori information about the lower and upper bound of the complex permittivity contrast, cf. (25) is not used.

\section{A. Synthetic Arm}

As a first example, we consider a synthetic model of human forearm. The data are collected using the measurement setup and frequency of operation as the ones used for the experimental data. The original profile of the synthetic human forearm is given in top plots of Fig. 7. The background medium is water 
(77.3 - j8.6). The model consists of bones with complex permittivity $5.5-j 0.59$, marrows with $8-j 0.98$, and four blood vessels with $63-j 14$. The permittivity of the muscle is $54-j 13$. The sizes of the object domains $D$ in Fig. 7 are $4.5 \lambda$ by $4.5 \lambda$.

In the inversion, this test domain $D$ is discretized into 45 by 45 cells. The inversion results from the TM-polarization measurement after 1024 iterations are given in the middle plots of Fig. 7. After 1024 iterations, the normalized error $F_{n}^{S}$ in the data equations and the contrast error $\mathrm{ERR}_{n}$ are reduced to $2.63 \%$ and $7.78 \%$, and adding more iterations does not improve or change the result. One iteration now takes approximately $8 \mathrm{~s}$.

The inversion results from the TE-polarization measurement after 1024 iterations are given in the bottom plots of Fig. 7. After 1024 iterations, the normalized error $F_{n}^{S}$ in the data equations and the contrast error $\mathrm{ERR}_{n}$ are reduced to $1.11 \%$ and $6.30 \%$, and adding more iterations does not improve the result. One iteration now takes approximately $16 \mathrm{~s}$. We observe that the boundaries of the bones are reconstructed better than the ones obtained from TM-polarization measurements. The reason for the improved reconstruction is based on the fact that in the interior of the test domain $D$ the electric fields are not tangential of the different tissue, so that the jumps in these components yield better reconstruction results of these interfaces. In order to investigate this improvement, we consider a more inhomogeneous example, namely the synthetic neck.

\section{B. Synthetic Neck}

The same measurement setup is still used as the previous example (64 sources with 33 receivers in front of a particular source), but now the radius of the antennas is $11.19 \mathrm{~cm}$ and the frequency of operation is $1 \mathrm{GHz}$. The complex permittivity of water at this frequency of operation is $78-j 3.6$. The original profile of this simple neck model is given in the top plots of Fig. 8. The neck model consists of fat tissue with complex permittivity $28-j 13.5$, cartilage with $25-j 10.78$, veins/arteries with $63-j 20$, bone with $6.4-j 2.16$, trachea 1 , and marrow $5.5-j 0.59$. The permittivity of the muscle is $50-j 23.37$. The size of the domain $D$ in Fig. 8 is $3.3 \lambda$ by $3.9 \lambda$ where $\lambda=$ is the wavelength in water at frequency of operation $f=1 \mathrm{GHz}$.

In the inversion, this test domain $D$ is discretized into 33 by 37 cells. The inversion results from the TM polarization measurement after 1024 iterations are given in the middle plots of Fig. 8. After 1024 iterations, the normalized error $F_{n}^{S}$ in the data equations and the contrast error $\mathrm{ERR}_{n}$ are reduced to $0.06 \%$ and $17.97 \%$, and adding more iterations does not improve the result. Note that, contrary to the results for the synthetic arm, the normalized errors in the data equations are very small, while the error in contrast is still large. This indicates a lack of information.

Next, we investigate whether we can improve the results using the TE-polarization measurements. The inversion results from the TE-polarization measurement after 1024 iterations are given in the bottom plots of Fig. 8. We observe that the results indeed improved. After 1024 iterations, the normalized error $F_{n}^{S}$ in the data equations and the contrast error $\mathrm{ERR}_{n}$ are reduced to $0.31 \%$ and $16.19 \%$, and adding more iterations does not improve the result.
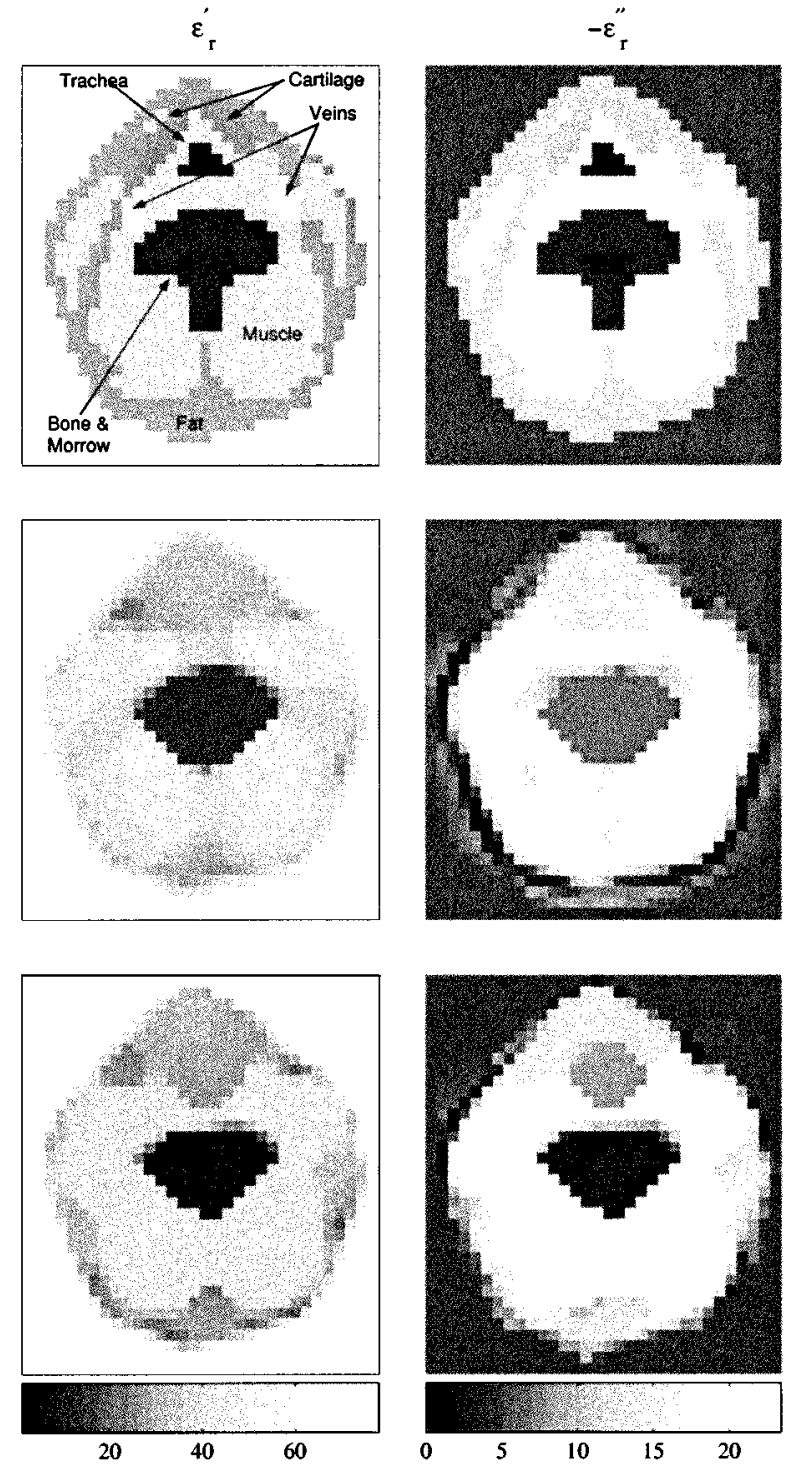

Fig. 8. Synthetic neck: images of the exact profile (top plots) and the results using 2-D TM polarization (middle plots), and using 2-D TE polarization (bottom plots).

\section{RESULTS OF FULL-VECTORIAL 3-D INVERSION}

As a test case for our full-vectorial 3-D inversion algorithm we use the 3-D version of the synthetic example in [18]. The measurement setup is similar to the one used by Bulyshev et al. [13] where they employ a scalar approximation of the field problem. We have three rings containing transmitter and receiver antennas. A schematic view of the transmitter-receiver setup and the test domain $D$ is given in Fig. 9. All receivers operate simultaneously (in all three rings) while the transmitter operates one after another. The receiver records all the components of the vector electric field $\boldsymbol{E}_{i}^{\text {sct }}$ (multicomponent receiver). The transmitter is modeled using a point magnetic dipole directed in the $i_{3}$ direction viz.

$$
\begin{gathered}
\boldsymbol{E}_{i}^{\mathrm{inc}}(\boldsymbol{x})=j \omega \mu_{0} \frac{\exp \left(-j k_{b}\left|\boldsymbol{x}-\boldsymbol{x}_{i}^{S}\right|\right)}{4 \pi\left|\boldsymbol{x}-\boldsymbol{x}_{i}^{S}\right|^{3}}\left(-j k_{b}\left|\boldsymbol{x}-\boldsymbol{x}_{i}^{S}\right|-1\right) \\
\cdot\left[\left(x_{2}-x_{2, i}^{S}\right) \boldsymbol{i}_{1}-\left(x_{1}-x_{1, i}^{S}\right) \boldsymbol{i}_{2}\right] .
\end{gathered}
$$




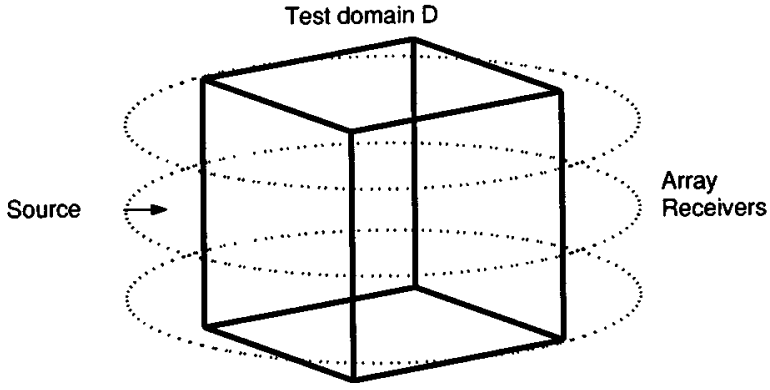

Fig. 9. Schematic of the antenna positions and the test domain $D$.
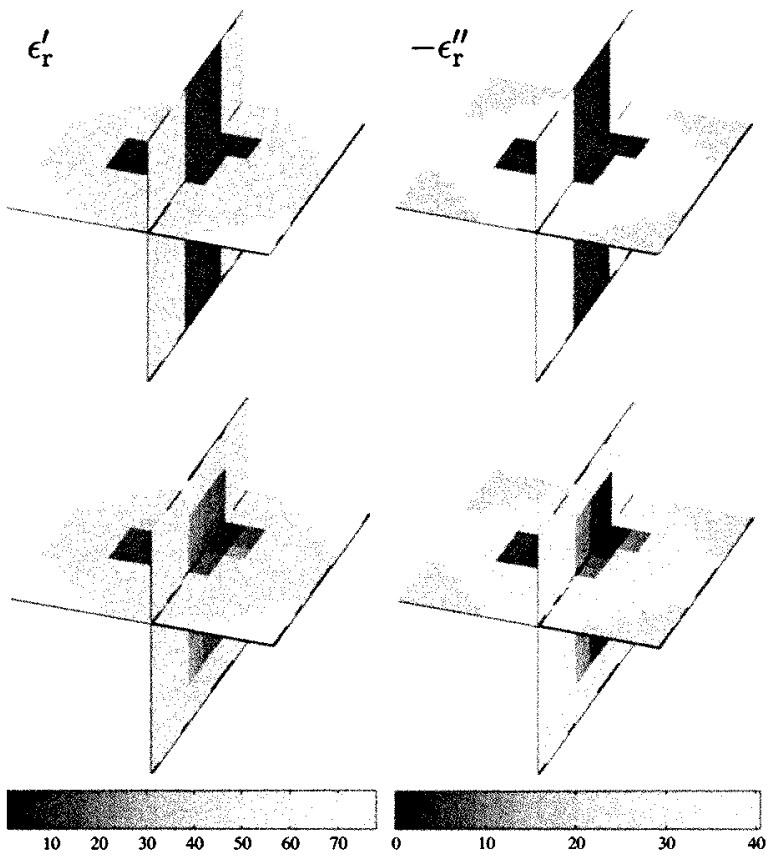

Fig. 10. Volume slices of the original profile (top plots) and the reconstructed results (bottom plots).

The frequency of operation of the transmitters is $f=1 \mathrm{GHz}$. In each ring, we have 6 transmitter stations and 30 receiver stations, thus in total we have $18 \times 90$ data points. The vertical positions of the circular rings are $x_{3}^{S}=-\lambda, 0$, and $-\lambda$, where $\lambda=$ $0.0333 \mathrm{~m}$, is the wavelength in water with complex permittivity $77.3-j 21.2$. Note that making the number of transmitters equal to the number of receivers could be extremely expensive from a computational point of view.

The original profile which has been used to generate synthetic data are given in the top plots of Fig. 10. These plots show the volume slices at $x_{1}=0$ and $x_{3}=0$. The dimension (domain $D$ ) of the profiles in Fig. 10 is $3 \lambda$ by $3 \lambda$ by $3 \lambda$. The objects have a complex permittivity close to that of biological tissue (bone $=8.0-j 3.2$ and muscle $=49.6-j 40.4$ ), and they are immersed in water. The synthetic data are generated by a conjugate gradient FFT method using discretization cells of $30 \times$ $30 \times 30$. The technique to obtain the discrete form of the algorithm is described in [19]. After generation of synthetic data, $5 \%$ random additive white noise is added.

In the inversion, we discretize the test domain $D$ into 15 by 15 by 15 cells, thus the number of complex unknowns is equal to 3375 . The reconstruction results after 1024 iterations are given
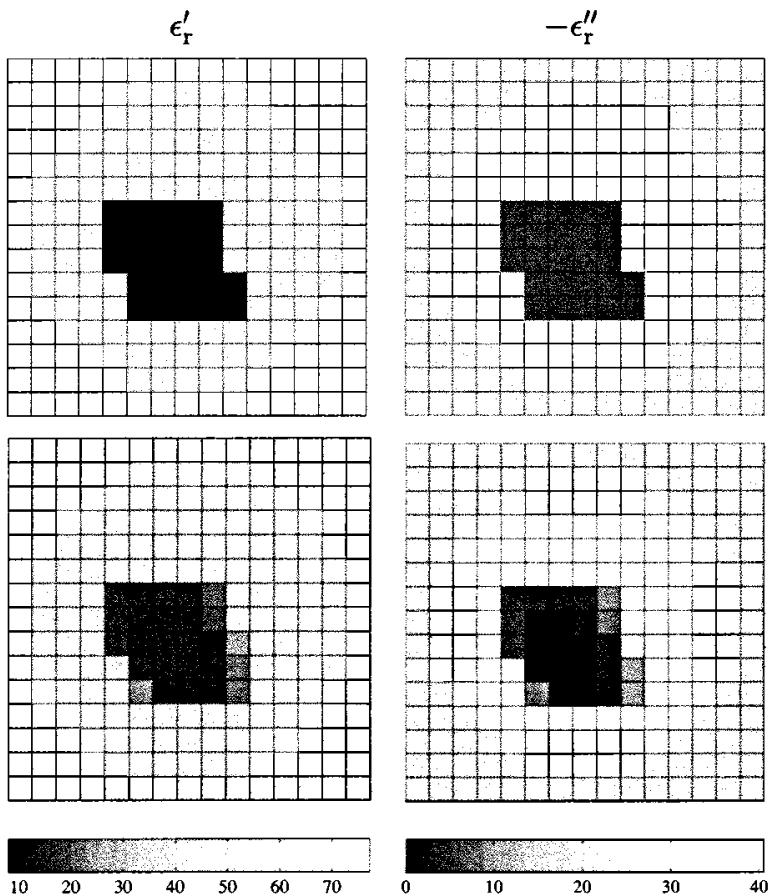

Fig. 11. Contour plots of the original profile (top plots) and the reconstructed results (bottom plots) at $x_{3}=0$.

in the bottom plots of Fig. 10. Now, one iteration takes approximately $22 \mathrm{~s}$ on a personal computer with a $600-\mathrm{MHz}$ Pentium III processor. We observe that the results are quite satisfactory in spite of the use of limited data. The only drawback is that the resolutions in the vertical direction are worse than those in the transversal plane. This is due to the limited number of transmitters and receivers in the vertical direction. In order to have more details on the reconstruction results, we present also the contour plots in Fig. 11 at $x_{3}=0$ (the plane in the middle ring).

\section{CONCLUSION}

The present results of experimental biomedical data using a 2-D TM-polarization measurement at $2.33 \mathrm{GHz}$ show that the MR-CSI method leads to an effective inversion technique. The algorithm is fully iterative and does not solve any forward problem in each of its iterative steps. This makes the method suitable for large scale computations. Furthermore, the artificial tuning process with a weighting parameter of the regularization to obtain the "cosmetically best" results seems superfluous.

From the feasibility study results of the synthetic arm and the synthetic neck of the 2-D TM- and 2-D TE-polarization measurements, we observe that the algorithm is not able to reconstruct tissues with a dimension less than half the wavelength. This limitation is known as the Rayleigh criterion. Thus, in order to image inhomogeneities with sizes less than half a wavelength, one has to use a higher frequency (shorter wavelength) wave field experiment. The problem with using a single high-frequency experiment is the increase of the nonlinearity of the problem, which will effect the performance of the algorithm. Thus, in order to robustly solve this problem, a multifrequency experiment inversion has to be included (see, e.g., [20]) and because the Maxwell model (due to the 
relaxation effect of the complex permittivity) no longer holds, a more complicated model, e.g., the Debye model, has to be used. Moreover, under the hardware point of view, some issues concerning the SNR, repeatability, and isolation requirements of the scanner must be investigated. This is one subject of our future research.

As our final test study, we show a simple example of inversion of full-vectorial 3-D biomedical media. The results indicate that the inversion method can obtain satisfactory 3-D results using a limited data set.

\section{APPENDIX}

The MR-CSI method starts with the updating of the contrast sources and contrast in the following manner.

\section{A. Updating the Contrast Sources}

Define the data error and the object error to be

$$
\begin{aligned}
& \rho_{i, n}=f_{i, n}-G_{S} w_{i, n} \\
& r_{i, n}=\chi_{n} u_{i}^{\mathrm{inc}}-w_{i, n}+\chi_{n} G_{D} w_{i, n} .
\end{aligned}
$$

Now suppose $w_{i, n-1}$ and $\chi_{n-1}$ are known. We update $w_{j}$ by

$$
w_{i, n}=w_{i, n-1}+\alpha_{n}^{w} v_{i, n}
$$

where $\alpha_{n}^{w}$ is a real constant parameter and the update directions $v_{i, n}$ are functions of position. The update directions are chosen to be the Polak-Ribière conjugate gradient directions. These update directions are given by

$$
\begin{aligned}
& v_{i, 0}=0 \\
& v_{i, n}=g_{i, n}^{w}+\frac{\operatorname{Re} \sum_{k}\left\langle g_{k, n}^{w}, g_{k, n}^{w}-g_{k, n-1}^{w}\right\rangle_{D}}{\sum_{k}\left\langle g_{k, n-1}^{w}, g_{k, n-1}^{w}\right\rangle_{D}} v_{i, n-1}
\end{aligned}
$$

where

$$
g_{i, n}^{w}=-\eta^{S} G_{S}^{\star} \rho_{i, n-1}-\eta_{n}^{D}\left[r_{i, n-1}-G_{D}^{\star}\left(\bar{\chi}_{n-1} r_{i, n-1}\right)\right] .
$$

In (32), $G_{S}^{\star}$ and $G_{D}^{\star}$ are the adjoints of $G_{S}$ and $G_{D}$ mapping $L^{2}(S)$ into $L^{2}(D)$ and $L^{2}(D)$ into $L^{2}(D)$, respectively. Further, the overbar denotes complex conjugate. The real parameter $\alpha_{n}^{w}$ in (30) is found explicitly to be

$$
\alpha_{n}^{w}=\frac{-\operatorname{Re} \sum_{i}\left\langle g_{i, n}^{w}, v_{i, n}\right\rangle_{D}}{\eta^{S} \sum_{i}\left\|G_{S} v_{i, n}\right\|_{S}^{2}+\eta_{n}^{D} \sum_{j}\left\|v_{i, n}-\chi_{n-1} G_{D} v_{i, n}\right\|_{D}^{2}} .
$$

We choose as starting values the contrast sources that minimize the normalized errors in the data equations $F^{S}$, which are the contrast sources obtained by back propagation

$$
w_{i, 0}^{b p}=\frac{\left\|G_{S}^{\star} f_{i}\right\|_{D}^{2}}{\left\|G_{S} G_{S}^{\star} f_{i}\right\|_{S}^{2}} G_{S}^{\star} f_{i} .
$$

\section{B. Updating of the Contrast}

Before updating the contrast, we first compute

$$
u_{i, n}=u_{i}^{\mathrm{inc}}+G_{D} w_{i, n}=u_{i, n-1}+\alpha_{n}^{w} v_{i, n} .
$$

Now, supposing that $w_{i, n}$ and $\chi_{n-1}$ are known, we update $\chi$ by

$$
\chi_{n}=\chi_{n-1}+\alpha_{n}^{\chi} d_{n}
$$

where $\alpha_{n}^{\chi}$ is a constant parameter and the update direction $d_{n}$ is taken as the Polak-Ribière conjugate gradient direction

$$
\begin{aligned}
& d_{0}=0 \\
& d_{n}=g_{n}^{\chi}+\frac{\operatorname{Re}\left\langle g_{n}^{\chi}, g_{n}^{\chi}-g_{n-1}^{\chi}\right\rangle_{D}}{\left\langle g_{n-1}^{\chi}, g_{n-1}^{\chi}\right\rangle_{D}} d_{n-1} .
\end{aligned}
$$

As in [12], the preconditioned gradient $g_{n}^{\chi}$ in (37) is given by

$$
g_{n}^{\chi}=\frac{\eta_{n}^{D} g_{n}^{D}+F_{n}\left(w_{j, n}, \chi_{n-1}\right) g_{n}^{R}}{\sum_{j}\left|u_{j, n}\right|^{2}}
$$

where

$$
g_{n}^{D}=-\eta_{n}^{D} \sum_{j}\left(\chi_{n-1} u_{i, n}-w_{i, n}\right) \bar{u}_{i, n}
$$

and

$$
g_{n}^{R}(\boldsymbol{x})=\frac{1}{V} \nabla \cdot\left[\frac{\nabla \chi_{n-1}}{\left|\nabla \chi_{n-1}\right|^{2}+\delta_{n-1}^{2}}\right] .
$$

Note that the gradient $g_{n}^{\chi}$ tends to the direction $g_{n}^{D}$ as the gradient $g_{n}^{R}$ tends to zero. The weighting of the gradients clearly depends on the normalized errors in the cost function $F_{n}$. Since we have a multiplicative cost function, one can expect a higher nonlinear functional, but the gradient of this cost function has the same form as the gradient of an additive cost function with a weighting parameter related to $F_{n}$; see, e.g., [9]. Moreover, we observe that the gradient of the present regularization factor is similar to the one of total-variation regularization; see [9]. Hence, this weighted $L^{2}(D)$ regularization factor combines the features of minimization of the total variation in the $L^{2}(D)$-norm and in the $L^{1}(D)$-norm (through its gradient).

The minimization of the multiplicative cost functional (16) can be performed analytically. The cost functional is a fourthdegree polynomial in $\alpha=\alpha_{n}^{\chi}$ viz.

$$
\begin{aligned}
C= & {\left[F^{S}\left(w_{i, n}\right)+F_{n}^{D}\left(w_{i, n}, \chi_{n-1}\right)+\alpha^{2} \eta_{n}^{D} \sum_{i}\left\|d_{n} u_{i, n}\right\|_{D}^{2}\right.} \\
& \left.+2 \alpha \eta_{n}^{D} \operatorname{Re} \sum_{i}\left\langle d_{n} u_{i, n}, \chi_{n-1} u_{i, n}-w_{i, n}\right\rangle_{D}\right] \\
\times & {\left[1+2 \alpha \operatorname{Re}\left\langle b_{n-1} \nabla \chi_{n-1}, b_{n-1} \nabla d_{n}\right\rangle_{D}+\alpha^{2}\left\|b_{n-1} \nabla d_{n}\right\|_{D}^{2}\right] }
\end{aligned}
$$

where

$$
b_{n-1}=V^{-1 / 2}\left(\left|\nabla \chi_{n-1}\right|^{2}+\delta_{n-1}^{2}\right)^{-1 / 2} .
$$


Differentiation with respect to $\alpha$ yields a cubic equation with one real root and two complex conjugate roots. The real root is the desired minimizer $\alpha_{n}^{\chi}$.

As far as the starting value $\chi_{0}$ is concerned, we start with the initial estimates $w_{i, 0}^{b p}$ of (34) and compute the initial field $u_{i, 0}$ using (35) to obtain

$$
\chi_{0}=\frac{\sum_{i} w_{i, 0}^{b p} \bar{u}_{i, 0}}{\sum_{i}\left|u_{i, 0}\right|^{2}}, \quad u_{i, 0}=u_{i}^{\mathrm{inc}}+G_{D} w_{i, 0}^{b p} .
$$

This completes the description of the MR-CSI algorithm.

\section{ACKNOWLEDGMENT}

The authors wish to express their gratitude to Prof. A. Broquetas and his research group for providing the various experimental data. These experimental data are available to the general community and can be obtained by contacting the authors.

\section{REFERENCES}

[1] J. C. Bolomey, C. Pichot, and G. Gaboriaud, "Planar microwave imaging camera for biomedical applications: Critical and prospective analysis of reconstruction algorithms," Radio Sci., vol. 26, pp. 541-549, 1991.

[2] P. M. van den Berg and R. E. Kleinman, "Contrast source inversion method," Inverse Problems, vol. 13, pp. 1607-1620, 1997.

[3] W. C. Chew and Y. M. Wang, "Reconstruction of two-dimensional permittivity distribution using the distorted Born iterative method," IEEE Trans. Med. Imag., vol. 9, pp. 218-225, June 1990.

[4] A. Franchois and C. Pichot, "Microwave imaging-Complex permittivity reconstruction with a Levenberg-Marquadt method," IEEE Trans. Antennas Propagat., vol. 45, pp. 203-215, Feb. 1997.

[5] A. Roger, "Newton-Kantorovich algorithm applied to an electromagnetic inverse problem," IEEE Trans. Antennas Propagat., vol. AP-29, pp. 232-238, 1981.

[6] J. J. Mallorqui, N. Joachimowicz, A. Broquetas, and J. C. Bolomey, "Quantitative images of large biological bodies in microwave tomography by using numerical and real data," Electron. Lett., vol. 23, pp. 2138-2140, 1996.

[7] N. Joachimowicz, J. J. Mallorqui, J. C. Bolomey, and A. Broquetas, "Convergence and stability assessment of Newton-Kantorovich reconstruction algorithms for microwave tomography," IEEE Trans. Med. Imag., vol. 17, pp. 562-569, Aug. 1998.

[8] A. Abubakar and P. M. van den Berg, "Three-dimensional nonlinear inversion in crosswell electrode logging," Radio Sci., vol. 4, pp. 989-1004, 1998.

[9] P. M. van den Berg, A. L. van Broekhoven, and A. Abubakar, "Extended contrast source inversion," Inverse Problems, vol. 15, pp. 1325-1344, 1999.

[10] A. Abubakar, P. M. van den Berg, and B. J. Kooij, "A conjugate gradient contrast source technique for 3D profile inversion," IEICE Trans. Electron., vol. E83-C, pp. 1864-1874, 2000.

[11] R. F. Bloemenkamp, A. Abubakar, and P. M. van den Berg, "Inversion of experimental multi-frequency data using the contrast source inversion method," Inverse Problems (Special Issue), vol. 17, pp. 1611-1622, Dec. 2001.

[12] P. M. van den Berg and A. Abubakar, "Contrast source inversion method: State of art," Progress Electromagn. Res., vol. PIER 34, pp. 189-218, 2001.

[13] A. E. Bulyshev, A. E. Souvorov, S. Y. Semenov, R. H. Svenson, A. G. Nazarov, Y. E. Sizov, and G. P. Tatsis, "Three-dimensional microwave tomography. Theory and computer experiments in scalar approximation," Inverse Problems, vol. 16, pp. 863-875, 2000.

[14] A. Broquetas, J. Romeu, J. M. Rius, A. R. Elias-Fuste, A. Cardama, and L. Jofre, "Cylindrical geometry: A further step in active microwave tomography," IEEE Trans. Microwave Theory Tech., vol. 39, pp. 836-844, May 1991.
[15] J. H. Richmond, "Scattering by a dielectric cylinder of arbitrary cross section shape," IEEE Trans. Antennas Propagat., vol. AP-13, pp. 334-341, 1965.

[16] A. P. M. Zwamborn and P. M. van den Berg, "A weak form of the conjugate gradient FFT method for two-dimensional TE scattering problems," IEEE Trans. Microwave Theory Tech., vol. 39, pp. 953-960, June 1991.

[17] B. J. Kooij and P. M. van den Berg, "Nonlinear inversion in TE scattering," IEEE Trans. Microwave Theory Tech., vol. 46, pp. 1704-1712, Nov. 1998.

[18] N. Joachimowicz, C. Pichot, and J. P. Hugonin, "Inverse scattering: An iterative numerical method for electromagnetic imaging," IEEE Trans. Antennas Propagat., vol. 12, pp. 1742-1752, Dec. 1991.

[19] A. Abubakar and P. M. van den Berg, "A total variation enhanced contrast source inversion method for three-dimensional profile reconstruction," in Proc. ECCOMAS 2000, Book of Abstracts, Barcelona, Spain, Sept. 11-14, 2000, CD-ROM, p. 19, p. 1132.

[20] W. C. Chew and J. H. Lin, "A frequency-hopping approach for microwave imaging of large inhomogeneous bodies," IEEE Microwave Guided Wave Lett., vol. 5, pp. 439-441, Dec. 1995.

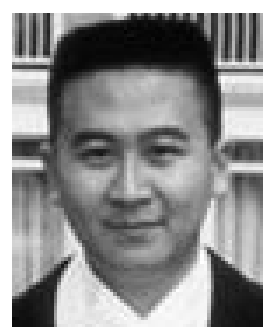

Aria Abubakar was born in Bandung, Indonesia, on August 21, 1974. He received the M.Sc. degree (cum laude) in electrical engineering and the Ph.D. degree (cum laude) in technical sciences from the Delft University of Technology, Delft, The Netherlands, in 1997 and 2000, respectively.

In 1996, he was a Research Student at Shell Research B.V., Rijswijk, The Netherlands. He was a Summer Intern with Schlumberger-Doll Research, Ridgefield, CT, in 1999. He is currently with the Laboratory of Electromagnetic Research and Section of Applied Geophysics, Delft University of Technology. His current main research activity includes solving forward and inverse problems in acoustics, electromagnetics, and elastodynamics.

Dr. Abubakar was the recipient of the Best 1997 Master's Thesis Award in electrical engineering given by Delft University of Technology.

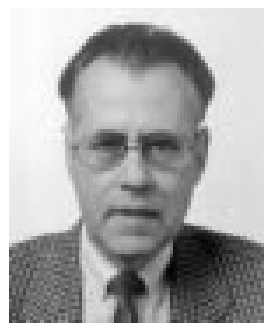

Peter M. van den Berg was born in Rotterdam, The Netherlands, on November 11, 1943. He received the degree in electrical engineering from the Polytechnical School of Rotterdam, Rotterdam, The Netherlands, in 1964, and the B.Sc. and M.Sc. degrees in electrical engineering and the Ph.D. degree in technical sciences from the Delft University of Technology, Delft, The Netherlands, in 1966, 1968 , and 1971, respectively.

From 1967 to 1968, he was a Research Engineer with the Dutch Patent Office. Since 1968, he has been a Member of the Scientific Staff of the Electromagnetic Research Group, Delft University of Technology. During these years, he carried out research and taught classes in the area of wave propagation and scattering problems. During the academic year 1973-1974, he was a Visiting Lecturer in the Department of Mathematics, University of Dundee, Dundee, Scotland, financed by an award from the Niels Stensen Stichting, The Netherlands. During a three-month period in 1980-1981, he was a Visiting Scientist at the Institute of Theoretical Physics, Göteborg, Sweden. He was appointed as a Professor at the Delft University of Technology in 1981. During the years of 1988-1994, he also carried out research at the Center of Mathematics of Waves, University of Delaware, Newark; these visits were financed by a NATO award. During the summer periods of 1993-1995, he was a Visiting Scientist at Shell Research B.V., Rijswijk, The Netherlands. Since 1994, he has also been a Professor in the Delft Research School Centre of Technical Geoscience. His current main research interest is the efficient computation of field problems using iterative techniques based on error minimization, the computation of fields in strongly inhomogeneous media, and the use of wave phenomena in seismic data processing. A major interest is in an efficient solution of the nonlinear inverse scattering problem. 


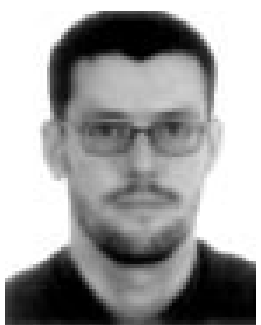

Jordi J. Mallorqui (S'91-M'93) received the Ingeniero and $\mathrm{Ph} . \mathrm{D}$. degrees in telecommunication engineering from the Universitat Politecnica de Catalunya (UPC), Barcelona, Spain, in 1990 and 1995, respectively, for his work on microwave tomography.

Since 1992, he has been with the Department of Signal Theory and Communications, UPC, as an Assistant and later Associate Professor. His research activities are concerned with electromagnetic numerical simulation and microwave imaging for biomedical and remote sensing applications. During 1999, he was with the Jet Propulsion Laboratory as a Guest Researcher where he developed calibration methods for airborne SAR interferometry. He is currently involved with the application of SAR interferometry to terrain subsidence monitoring and 3-D simulation of microwave tomography scanners. 